\title{
A corpus search methodology for focus realization
}

\section{Project Goals}

- Study phonetic realization of focus in cases where

formal semantic theories make predictions

- Develop and apply methodology for accessing controlled and quantifiable, but diverse and naturally occurring speech data

\section{Focus in Comparative Clauses}

- Coherent syntactic-semantic theory about focus placement

- Possibilities constrained: main clause usually antecedent for focus interpretation in comparative clause

\section{Subject focus}

(I) He stayed longer than [l] $]_{\mathrm{F}}$ did.

-er [[he stayed $x$ long $]_{2}$ than [I stayed $x$ long $] 2$

\section{Non-subject focus}

(2) I should have like that song more than I [did] F more $\lambda x[$ [should $\lambda w$ [I like that song $x$ well in $\mathbf{w}]]_{3}$

than [I like that song $x$ well in $\mathbf{w}_{\mathbf{0}}$ ]

(3) I understand even less than I did [before]F. even less [[I pres understand $x$ much $]_{4}$ then $[I$ understood $x$ much before $]] \sim 4$

\section{Corpus search methodology}

\section{- everyzuno .com}

- commercial search engine

- corpus of podcasts in mp3 format

- transcription and indexing with BBN speech recognition

- Web harvest

- Retrieval workflow

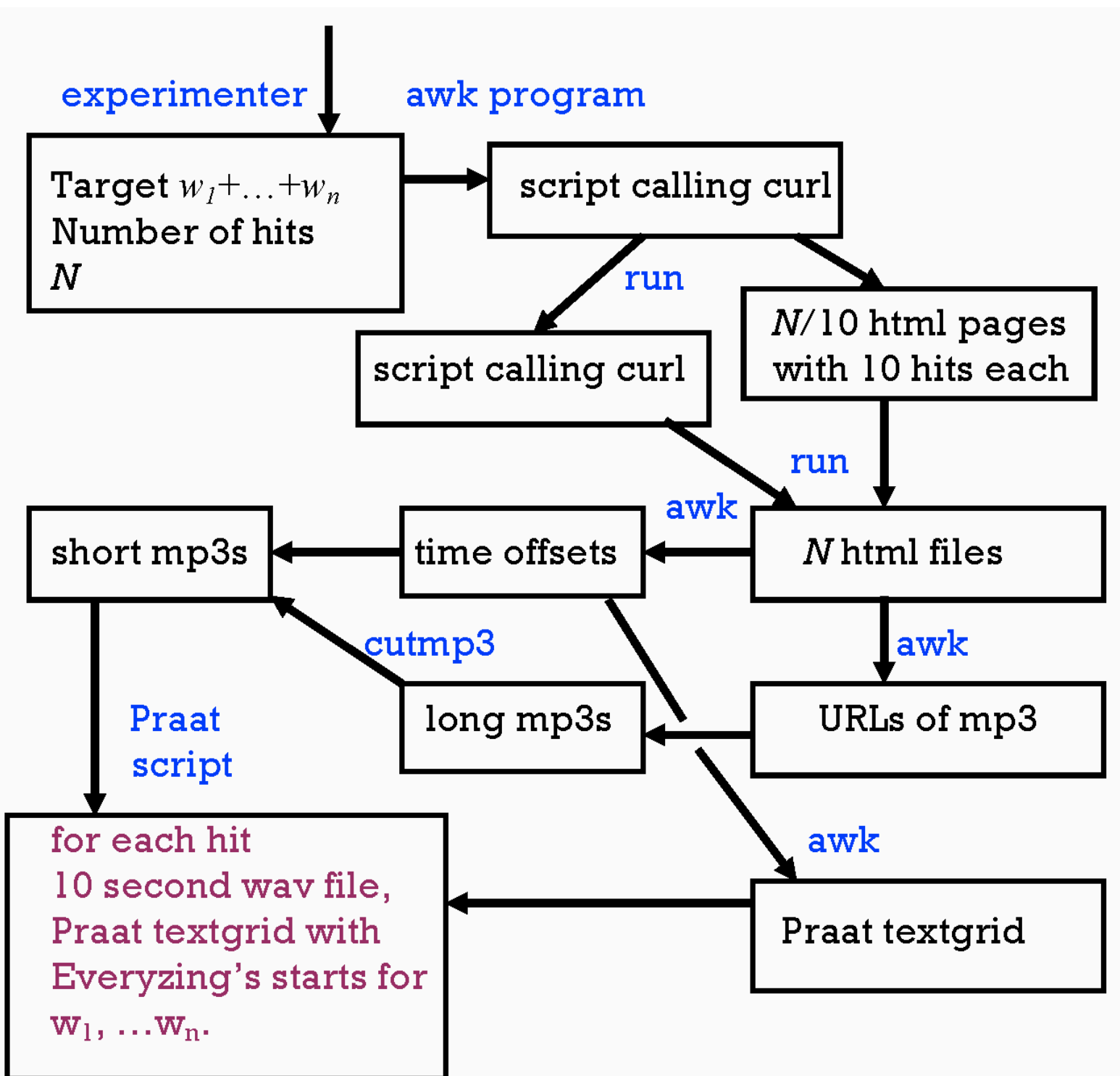

\section{Jonathan Howell}

Department of Linguistics

\section{Mats Rooth}

Department of Linguistics

Computing and Information Science

\section{Experiment: "than I did"}

\section{-Web harvest}

- I79 alleged tokens of "than I did"

- of which 91 unique correct tokens (56\%)

- Subphonemic annotation

- Experimenter annotation using landmarks

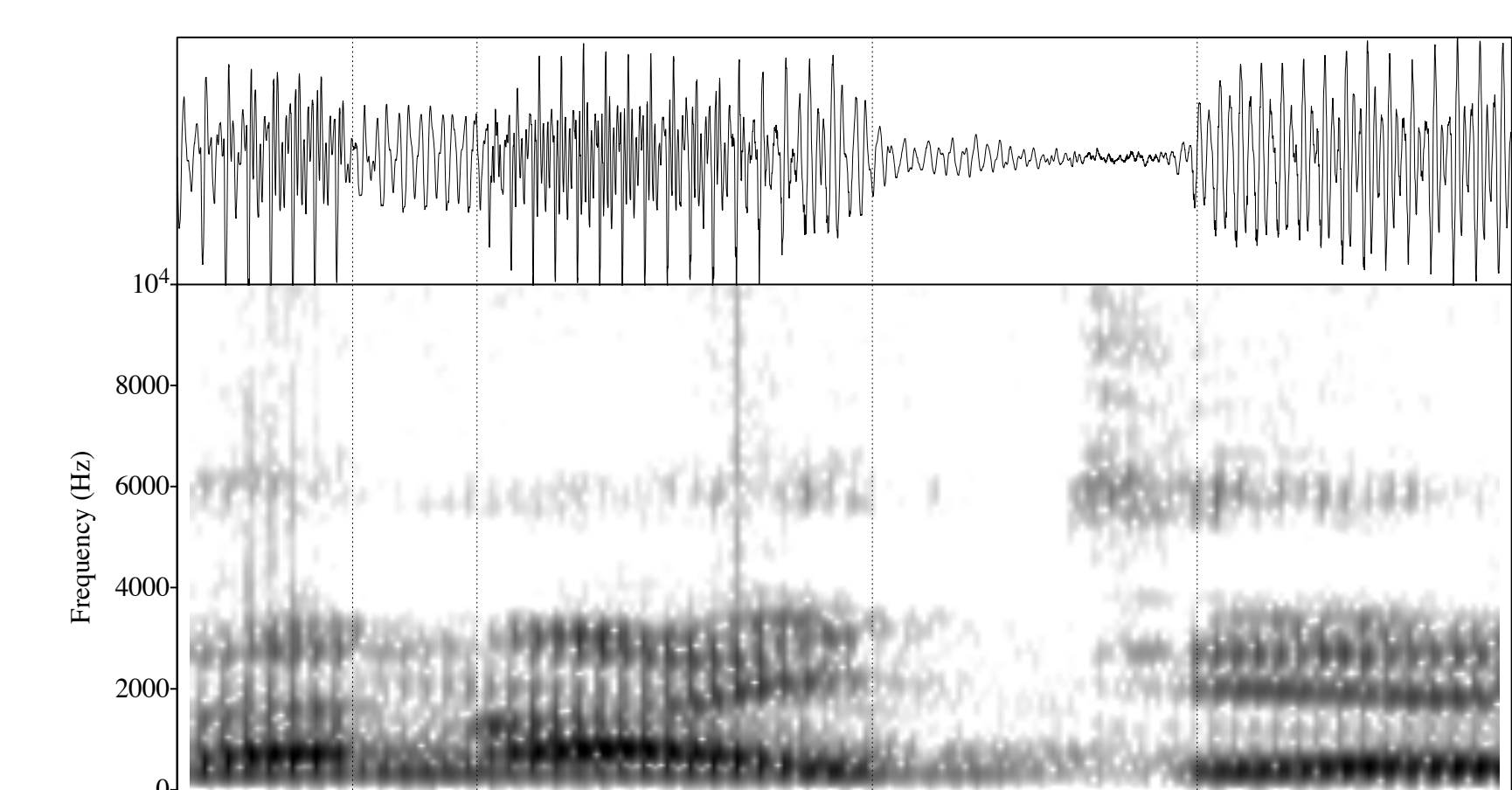

- Syntactic-semañtic clăssificătion

- Transcription into English prose

- Experimenter binary classification into subject focus condition "s" and non-subject focus condition "ns"

\begin{tabular}{|l|c|}
\hline \multicolumn{1}{|c|}{ Transcription } & Class \\
\hline [The aquarium got more attention] than [Ilid]. & $\mathrm{s}$ \\
\hline
\end{tabular}

\section{- Phonetic feature extraction}

- automated with Praat scripts

- 308 acoustic measures

Quantity: F0, amplitude, intensity, power...

Quality: formants, bandwidth, tilt, jitter.

Duration: for vowels, stops, syllables

- Measures taken: mean, extrema, range

- Locus of measure: regular intervals, time of other extrema (e.g. f0 max, intensity max)

- Supervised machine learning

- many parameters highly correlated (collinearity condition number $\mathrm{K}=127$ )

- want a focus detector for classifying novel/ controversial data

- SVM (support vector machine) increasingly common in machine learning

- SVM uses separating hyperplanes (margins) rather than comparison of mean

\section{- Comparing statistical classifiers}

- Classifiers evaluated by one-held-out cross validation (OHOCV)

- Best measures for each category (using feature selection techniques)
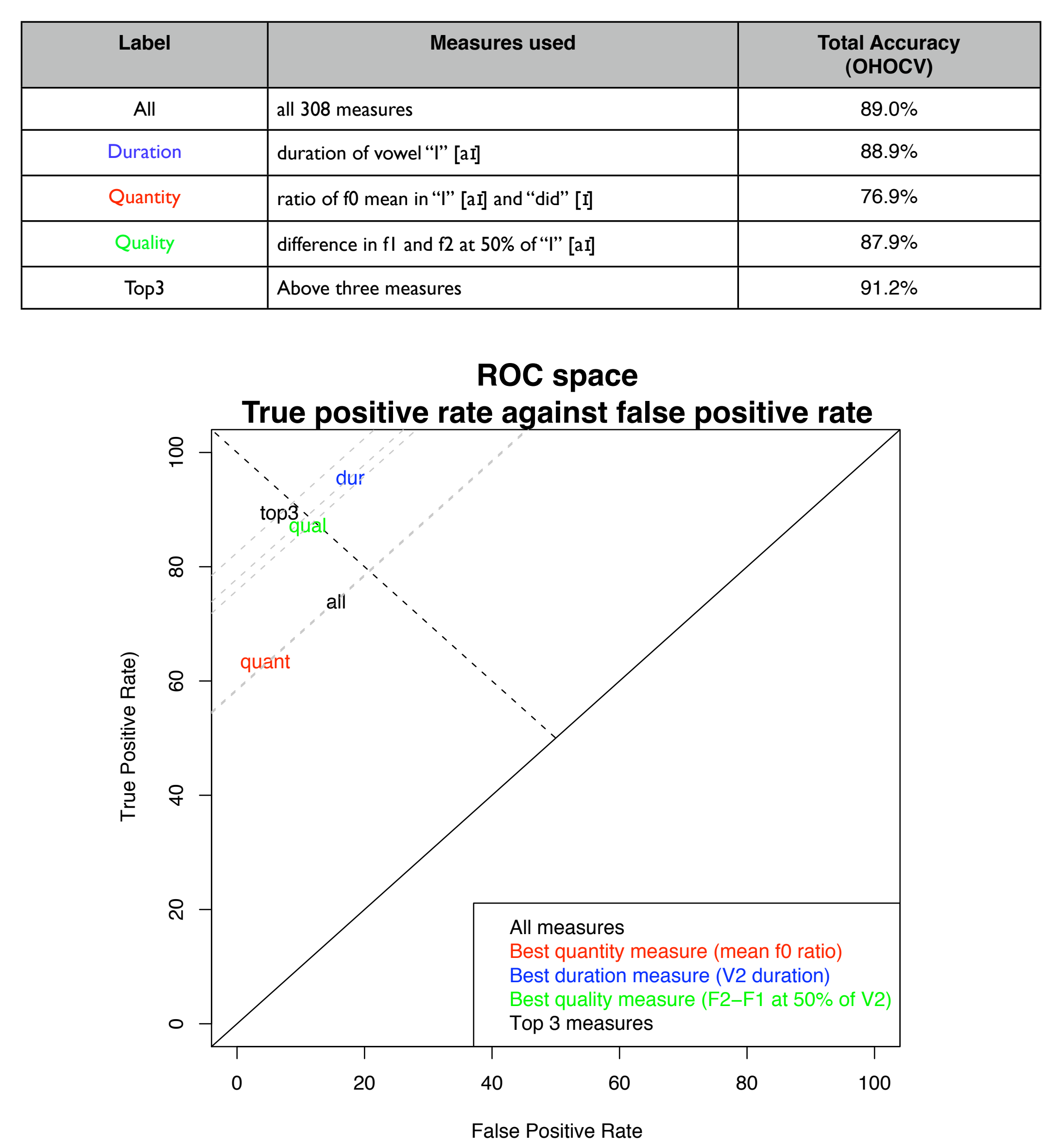

- Best-performing classifiers use features predictable from formal linguistic theory

- Best-performing quality measure outperforms best-performing quantity measure

- Comparing human classifiers

- Informal forced-choice discrimination task

"s" condition vs. "ns" condition

- Stimuli: 9l "than I did" snippets

- Non-naïve listeners $(n=5)$

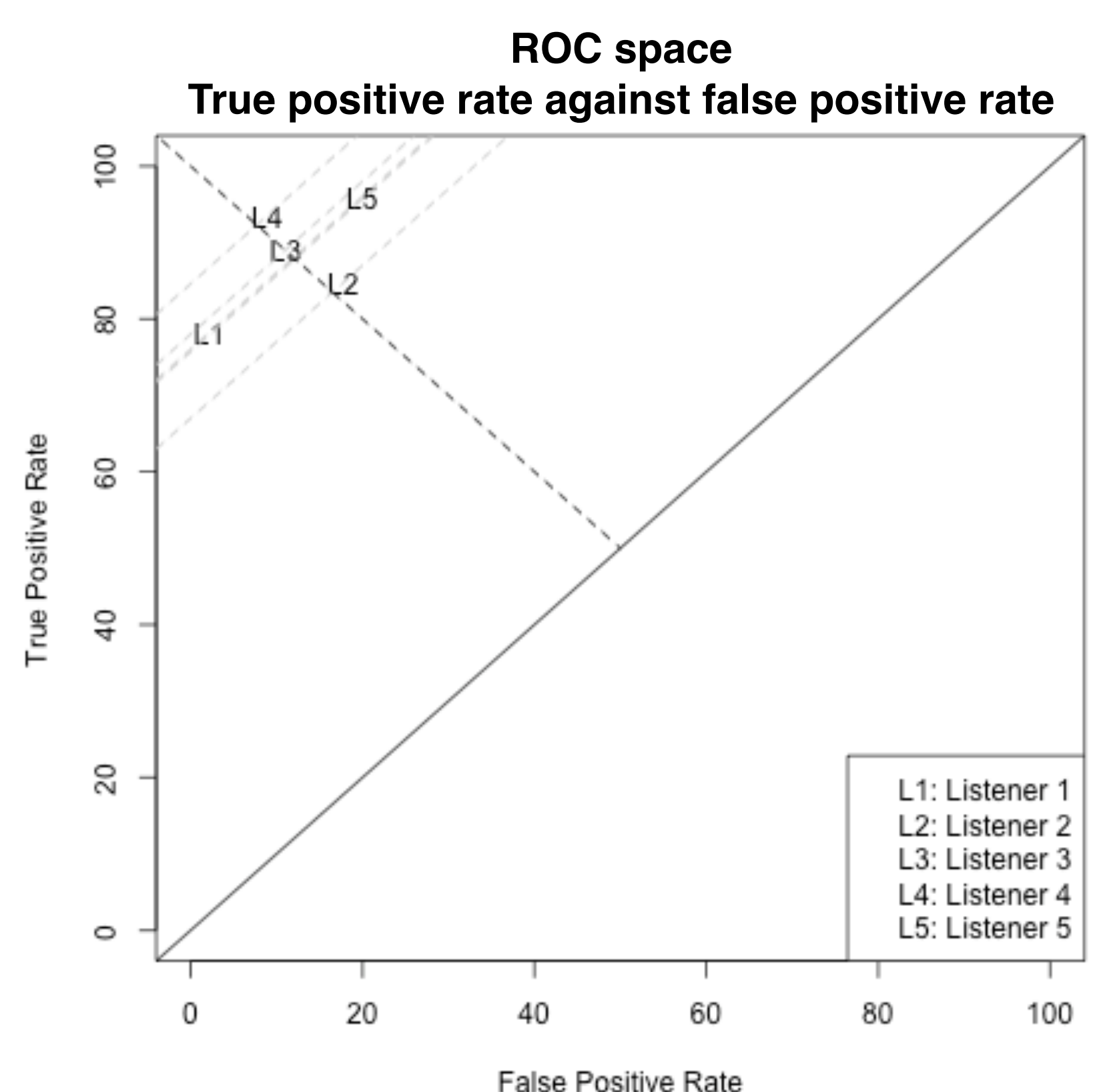

Phonetic models of accent:

hyperarticulation vs, sonority expansion

- Hyperarticulation / featural enhancement e.g. de Jong (1995), Fowler (1995), Cho (2005)

- vowel features or gestural targets maximized

- Sonority expansion / tongue or jaw lowering

e.g. Edwards \& Beckman (1988), Beckman et al. (1992), Erickson (2002)

- lower jaw / more open vocal tract

Predictions

\begin{tabular}{|c|c|c|}
\hline & Featural enhancement & Sonority expansion \\
\hline$[\mathrm{a}(\mathrm{I})]$ & $\begin{array}{l}\text { [low]: higher f1 } \\
\text { [back]: lower f2 }\end{array}$ & higher f1 \\
\hline [(h)i] & $\begin{array}{l}\text { [high]: lower f1 } \\
\text { [front]: higher f2 ? }\end{array}$ & higher f1 \\
\hline$[(w) i]$ & $\begin{array}{l}\text { [high]: lower f1 } \\
\text { [round]: lower f2? }\end{array}$ & higher f1 \\
\hline
\end{tabular}

Preliminary results consistent with featural enhancement

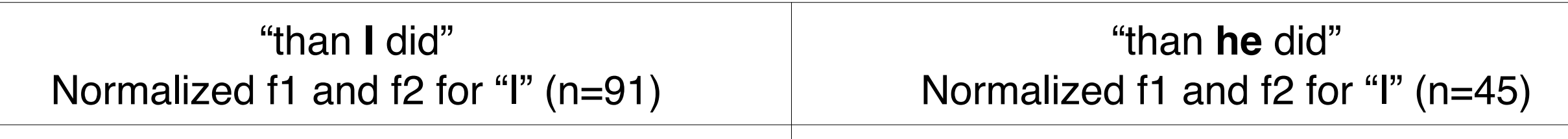
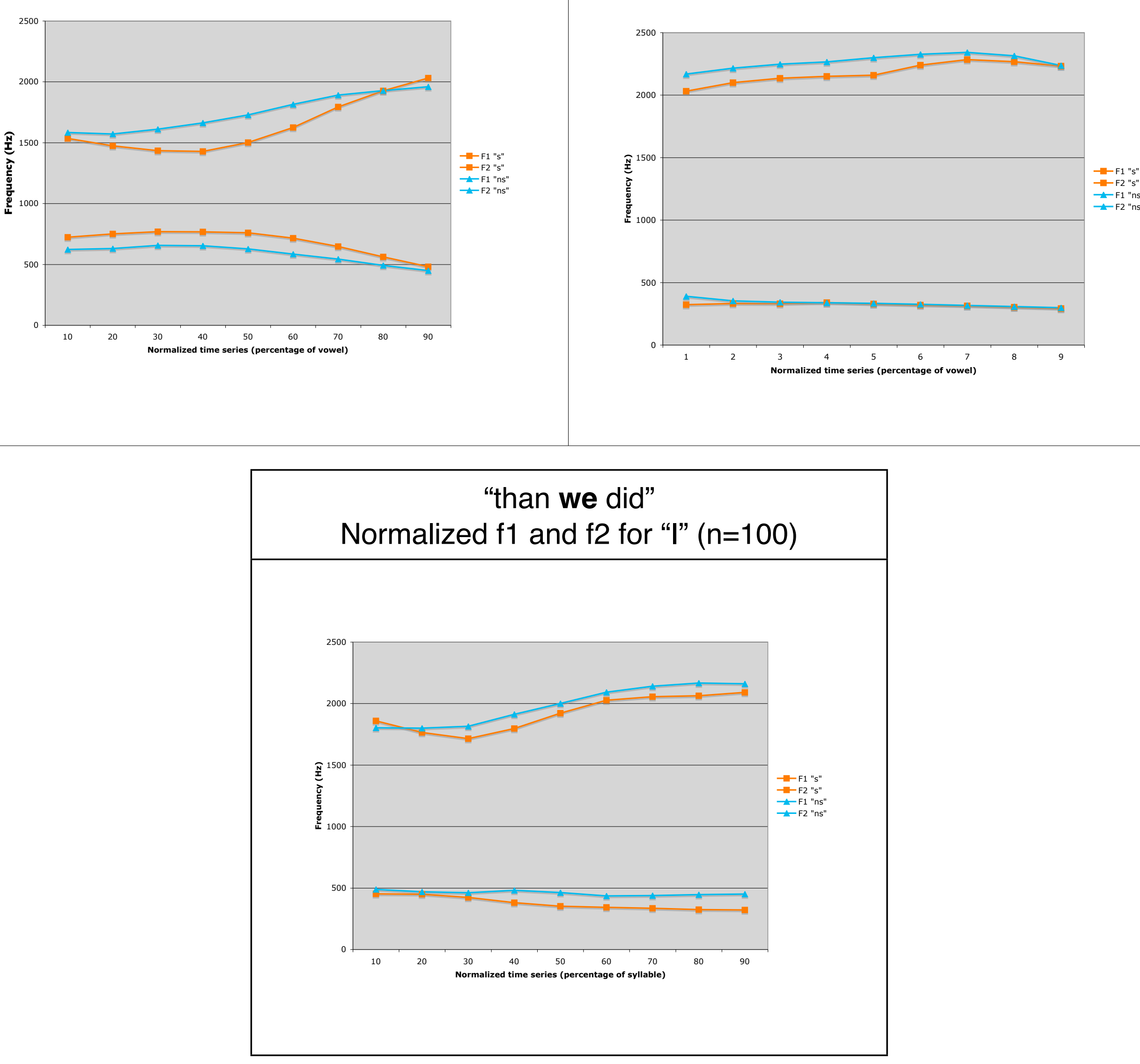
